\title{
Breast Cancer Identification Using Logistic Regression
}

\author{
S. Sathyavathi ${ }^{1}$, S. Kavitha ${ }^{2}$, R. Priyadharshini ${ }^{3}$ and A. Harini ${ }^{4}$ \\ ${ }^{1,2}$ Assistant Professor, Department of Information Technology, Kumaraguru College of Technology \\ ${ }^{3,4}$ Student, Department of Information Technology, Kumaraguru College of Technology.
}

\begin{abstract}
Best Cancer is a direct result of uncontrolled chest cell advancement. It happens in females and sometimes in folks. It is the second most compelling motivation for death from harmful development in women around the globe. The likelihood of a woman passing on from chest dangerous development is around 1 of each 38 (around 2.6\%). Since 2007, passing rates from chest threatening development have remained steady in women more energetic than 50, anyway have started to diminish in more prepared women. The death rate decreased by 1.3 percent consistently some place in the scope of 2013 and 2017. Dangerous development cells fill either in the lobules or in the chest courses. Lobules are milk-making organs, and lines are channels that pass on the milk to the areola from the organs. In this article, we intend to prescribe an approach to manage the assurance of chest threat subject to a collection of data factors portraying a couple of characteristics of sickness cell. This method uses a model called Logistic Regression for AI. Preliminary revelations show that the backslide model proposed is quantifiably critical and has higher precision.
\end{abstract}

KEY WORDS: BREAST CANCER, LOBULES, DUCTS, MACHINE LEARNING, LOGISTIC REGRESSION.

\section{INTRODUCTION}

Breast Cancer is a disorder in which breast cells develop out of control. There is a couple of breast cancer forms. The type depends on which cells transforms into cancer in breast. Breast cancer accounts for 25 percent of all cancer cases diagnosed in women as a worldwide figure from IARC (Indian Astrobiology Research Center). Around 53 percent of these cases come from developed countries, which account for 82 percent of the global population. Approximately 276,480 new cases of invasive breast cancer are predicted in 2020.Machine Learning has become a critical part of research in medical imaging. Over the years, Machine Learning approaches have progressed from manual seeded inputs to today's

\section{ARTICLE INFORMATION}

*Corresponding Author: Sathyavathi.s.it@kct.ac.in Received 10th Oct 2020 Accepted after revision 25th Nov 2020 Print ISSN: 0974-6455 Online ISSN: 2321-4007 CODEN: BBRCBA

Thomson Reuters ISI Web of Science Clarivate Analytics USA and Crossref Indexed Journal

\section{Clarivate
Analytics}

NAAS Journal Score 2020 (4.31) SJIF: 2020 (7.728)

A Society of Science and Nature Publication,

Bhopal India 2020. All rights reserved.

Online Contents Available at: http//www.bbrc.in/

Doi: http://dx.doi.org/10.21786/bbrc/13.11/8 automated initialization. As the learning ability of machine learning methods is continuously improving, developments in the field of machine learning have led to more intelligent and self -reliant computer assisted diagnosis (CAD) systems. We have proposed the logistic regression method to predict whether the patient has a malignant or benign tumor based on attribution.

Diagnosis during the early stages of life significantly enhances the future of women with breast cancer, by allowing for therapy as the cancer is rapidly developing. Machine learning is an application that gives systems the capability to learn and develop automatically from knowledge without being specifically programmed. Machine learning offers smart alternatives to the study of large data volumes. Machine Learning can generate precise results and analysis by designing quick and efficient algorithms and data-driven models for realtime data processing. In major field of machine imaging machine learning is widely used techniques in the prediction of the cancer diagnosis. 


\section{MATERIALS AND METHODS}

To conduct a series of experiments, publicly available breast cancer dataset is used .The following are the steps involved : Loading the dataset, Data preprocessing, Splitting the data into test and train, applying logistic regression to objects, evaluating accuracy.

Dataset collection: The dataset is considered from publicly available Kaggle website. The characteristics are determined from a digitized breast mass image the defines the characteristics of the nuclei of the cells present in the image. There are 569 rows and 33 columns in it. For each cell nucleus, the attributes are ID :number, diagnosis (M1=malignant,B1=benign)and ten real valued features are determined for each nucleus.

Data Pre-Processing: The function .info() from the pandas library is helpful to understand the basic properties of data fed. If there are any missing values in the data set, they can be identified and can be preprocessed before fitting into a model for training and perform testing .Preprocessing of data is an integral step as the quality of information and the valuable information that can be extracted from it directly affects our model to learn. The following unnecessary.

\section{features are dropped:}

ID: Which cannot be used for classification Class -Label: Diagnosis column

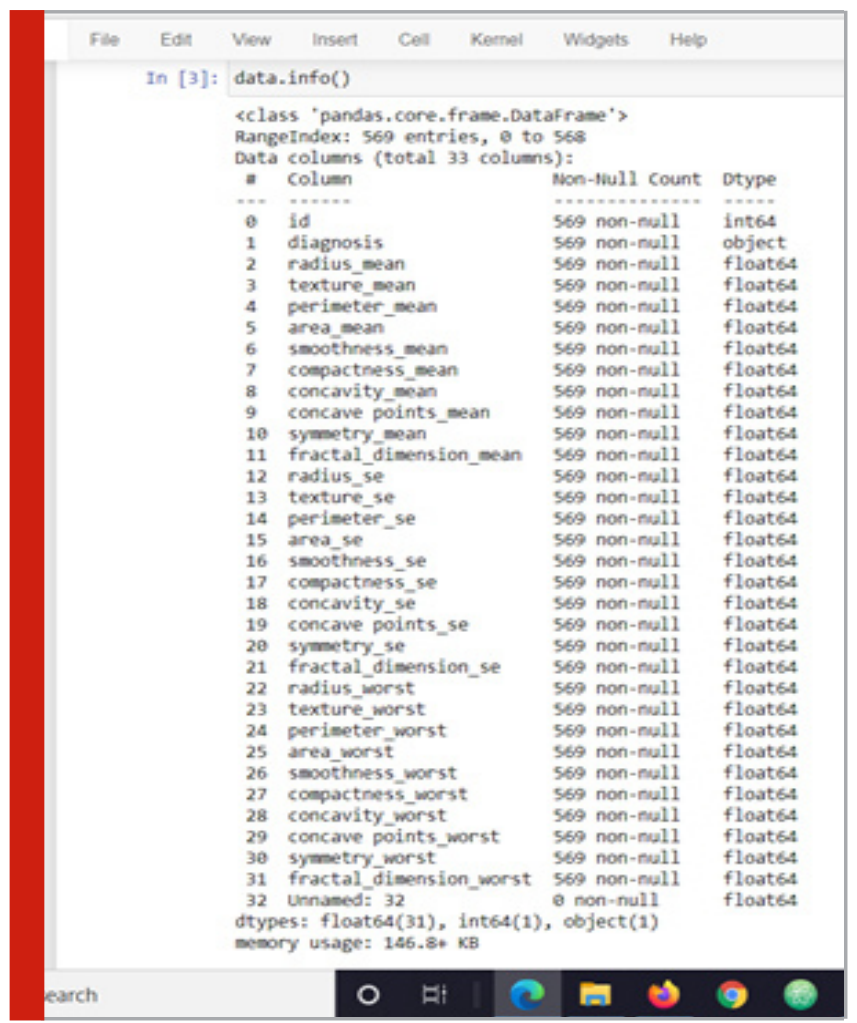

Unnamed Not applicable features: The pre-processed data after removing the missing values, replacing some default values, and preparing them for the training purpose. The following figure illustrates sample features or attributes the are considered after the preprocessing step and providing the accurate data attributes for the training the model with the actual data for the prediction.

Data Splitting: The data is split into train and test data in the ratio 80:20 to check the performance of the trained model. We used sci-kit learn open source machine learning library and imported "train-test split" from "sklearn_model selection" which splits array or matrices into random train and test subsets. The figure below gives the count of sample train and test data set considered for prediction.

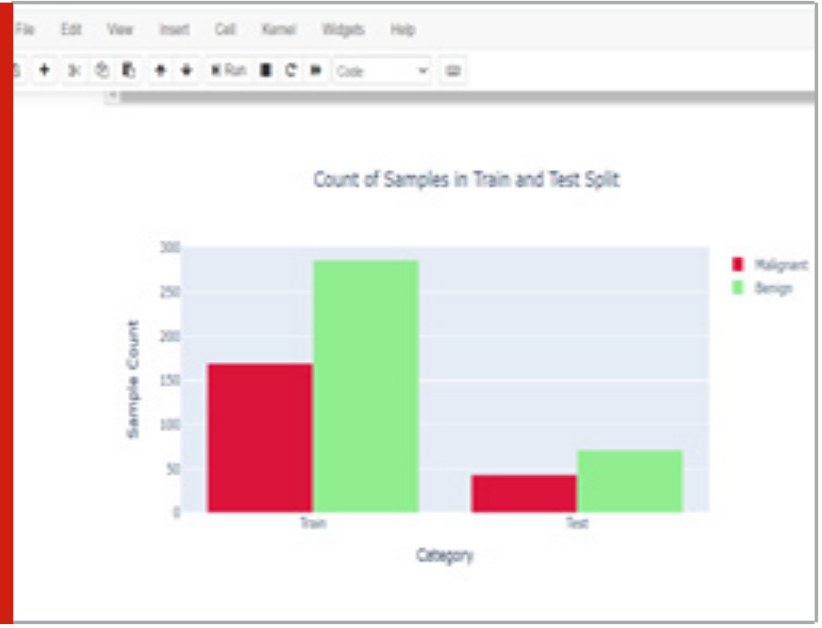

Normalization: Normalization is rescaling of real -valued numeric attributes into 0 and 1 range. Data normalization is implemented in machine learning in training less sensitive to the scale of the features. We have used MinmaxScaler() from the sklearn library for the normalization operation.

Logistic Regression: A popular machine learning algorithm used for classification is logistic regression. It is a statistical model and uses a logistic function to model a binary dependent variable in its basic form. The probability of an observation belonging to a certain class or classification is expected. Logistic regression converts the paradigm of linear regression into classifier and different types of regularization. The most common type of regularization methods are Ridge and Lasso. These two popular methods prevent overfitting. The technique of regularization is used to solve the overfitting issues by penalizing the cost function. The two regularization techniques used for processing are L1 or Lasso regularization and L2 or Ridge regularization.

Hypothesis: Our hypothesis "h1" should satisfy the following condition:

$$
\begin{gathered}
0<=\mathrm{h} 1(\mathrm{x})<=1 \\
\mathrm{~h} 1(\mathrm{x})=\mathrm{s} 1\left(\mathrm{w} 1 \mathrm{t}^{1 *} \mathrm{x}\right)
\end{gathered}
$$

where $\mathrm{x}$ is an observation, $\mathrm{s} 1$ is sigmoid function, $\mathrm{t} 1$ is time interval and w1 is weights. 
COST FOR AN OBSERVATION:

Case $0: \mathrm{h} 1(\mathrm{x})$ try to obtain results that are close to 0 as possible

Case 1: $\mathrm{h} 1(\mathrm{x})$ try to obtain results that are close to 1 as possible

\section{REGULARIZATION}

L2 regularization is used for the classification model.

The new cost function will be:

$$
\mathrm{C}(\mathrm{w} 1)=1 / \mathrm{n} \sum_{{ }_{\mathrm{i}}}^{\mathrm{n}}=1 \underset{\sum \mathrm{nj}=1 \mathrm{w} 1^{2}{ }_{\mathrm{j}}}{\operatorname{Cost}\left(\mathrm{h}\left(\mathrm{a}^{(\mathrm{i})}\right), \mathrm{b}^{(\mathrm{i})}\right)+\text { lambda } / 2 \mathrm{n}}
$$

The regularization term will heavily control the growth of $\mathrm{w} 1$. The $\mathrm{h} 1(\mathrm{x})$ we obtain with these controlled parameters w1 will be more generalizable. Also, the "lambada "is a hyper-parameter value and found out over cross validation.

If lambada is greater, it may lead to underfitting If lambada is equal to 0 , then there is no regularization effect. Thus, while choosing lambada ,it should be taken care so that the balance for bias vs variance trade -off is balanced properly.

Logistic Regression Parameters: Learning rate: For the advancement calculation (Gradient Descent), it is a tuning boundary that characterizes the progression at every cycle while moving towards a least clock work

Max_iter: Maximum number of iterations taken for the optimization algorithm to converge

Penalty: To perform L2 regularization

Tolerance: Value showing the weight between ages in which angle drop to be ended

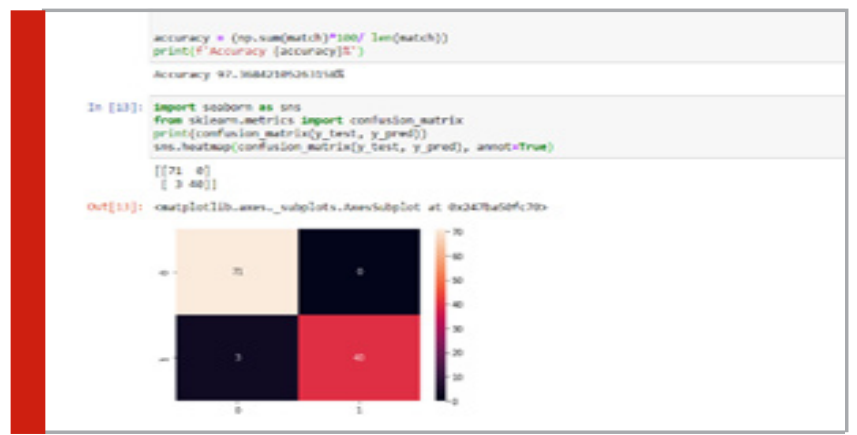

The subsequent stage is fitting the model as per the training data. The samples are taken to perform probability estimation and class label prediction operation. About 200 iterations are performed on the training data to be trained with logistic regression.

\section{RESULTS}

The confusion matrix, also called as error matrix is a particular table structure in the field of statistical classification. The table structure provides the visualization of the performance of the implemented function. Finally, the accuracy is measured and the confusion matrix is plotted using seaborn and sklearn metrics. The result is as follows: Thus, the accuracy obtained is $97.63 \%$.

\section{CONCLUSION}

In this work we led a progression of examination based on the machine learning models to improve breast cancer classification for the given data set. We have indicated that logistic regression method has applied on the training dataset shows the promising results. Our model achieves the accuracy of 97.63\%. In future work increased data in the data set can be provided and accuracy can be improved.

\section{REFERENCES}

Aishwarya Thangaraju Analysis of classification technique for medical data International journal of advanced research trends in engineering and technology vol 5 special issue 12

Bone D et al., (2015) Applying Machine Learning to Facilitate Autism Diagnostics: Pitfalls and Promises, J Autism Dev Disorf 45(5), pp.1121-1136.

Dua D and Graff C (2019) UCI Machine Learning Repository [http://archive.ics.uci.edu/ml]. Irvine, CA: University of California, School of Information and Computer Science.

F. A. Spanhol, L. S. Oliveira, C. Petitjean, and L. Heutte, (2016)“A Dataset for Breast Cancer Histopathological , "A Dataset for Breast Cancer Histopathological Image Classification," IEEE Transactions on Biomedical Engineering, vol. 63, no. 7, pp. 1455-1462

Ganesh N Sharma Rahul Various types and management of breast cancer: An overview Journal of advanced pharmaceutical technology and research 1(2) 109-126 Htet Thazin Tike Thein(2015) An approach for Breast cancer diagnosis classification using Neural network, Advanced computing An international Journal 6(1):111

Konstantina Kourou (2015) Machine learning applications in cancer prognosis and prediction ,Computational and structural Biotechnology journal Volume 13 pages 8-17

Seigel RL Miller KD Jemal(2016) A cancer statistics ,2016 A cancer journal for clinicians 2016 :66 (1):7-30 